\title{
Stationarity test of electromyographic signals during isokinetic trunk exertions
}

\author{
Young-Jin Cho a and Jung-Yong Kim ${ }^{\mathrm{a},}{ }^{*}$ \\ ${ }^{a}$ Department of Industrial and Management Engineering, Hanyang university, Sa 3-dong, Sangrok-gu, Ansan-si, \\ Gyeonggi-do, Korea
}

\begin{abstract}
The aim of this study is to investigate the stationarity of the electromyographic (EMG) signals during isokinetic exertions of the trunk muscle. Six healthy subjects participated in this study. The erector spinae muscles were used to collect the signals. In order to simulate the isokinetic exercises of the trunk, a Cybex system with a trunk modular component was used. All subjects performed sustained exertions with a combination of load $(25 \%, 50 \% \mathrm{MVC})$ and angular velocity $(0,15,30$ degree/s). The stationarity of the signals was tested by the reverse arrangements test and modified reverse arrangements test. In the results, the signal stationarity was high in all conditions in the reverse arrangements test. However, in the modified reverse arrangements test it was relatively low at about the 30 degree/s angular velocity. In the analysis of variance, the effect of angular velocity was statistically significant at the 0.05 significance level in the modified reverse arrangements test and Tukey's HSD grouping showed that the stationarity of 30 degree/s angular velocity was significantly different from 0,15 degree/s condition. These results indicate that the stationarity of the EMG signals showed no difference between isometric and isokinetic conditions when the angular velocity was equal to 15 degree/s.
\end{abstract}

Keywords: lifting, spectral analysis, low back pain, muscle fatigue

\section{Introduction}

Local muscle fatigue is an important cause of low back pain (LBP). In order to quantitatively assess muscle fatigue, it is necessary to analyze the frequency information of electromyographic (EMG) signals by using the spectral analysis. Fourier transform technique is widely used for frequency analysis. However, this technique is based on a mathematical assumption of signal stationarity $[2,8]$.

In General, EMG is stationary signal during isometric muscle contraction whereas nonstationary during dynamic contraction. However, signal stationarity has been shown to vary depending on experimental and analytical conditions such as load, window size and contraction types. EMG signals were stationary with $500 \mathrm{~ms}$ and $1000 \mathrm{~ms}$ sampling window sizes during $50 \%$ maximum voluntary contraction (MVC) from the biceps brachii muscle [6]. Bilodeau et al. (1997) reported that $93 \%$ of EMG signals were stationary when a $512 \mathrm{~ms}$ window size was used at $20-80 \%$ MVC from the biceps brachii muscle [1]. For the erector spinae muscle, Kim et al. (2005) showed that $94.55 \%$ of EMG signals were stationary at $15,30,45,60$ and $75 \%$ MVC during isometric contraction with 500-ms window size [7]. Coorevits et al. (2008) also reported that the stationarity of EMG signals were $91.6 \%$ with $256-\mathrm{ms}$ window size [4]. Cho and Kim (2010) investigated the stationarity of EMG signals during simulated lifting tasks with external loads and various flexion angles [3]. They reported that the stationarity of EMG signals were $99 \%$ in the reverse arrangements test and $88 \%$ in the modified reverse arrangements test.

Although there has been much research on the stationarity of the EMG signals, no researches had been conducted to examine the stationarity of trunk muscle EMG during isokinetic exertions. Therefore, the aim of this study is to investigate the EMG signal stationarity under various isokinetic trunk exertion conditions.

\footnotetext{
*Corresponding author. E-mail: jungkim@hanyang.ac.kr
} 


\section{Methods}

\subsection{Subjects}

Six healthy male subjects participated in this study. They had no history of low back disorder. Their height was a mean value of $175.0 \mathrm{~cm}$ with a $3.1 \mathrm{~cm}$ standard deviation. Their weight was a mean value of $69.2 \mathrm{~kg}$ with a $7.5 \mathrm{~kg}$ standard deviation.

\subsection{Apparatus}

The Humac Norm Testing and Rehabilitation with trunk module was used to simulate the isokinetic lifting task as shown in Figure 1. In order to collect electromyographic signals from the erector spinae muscle, ME6000 developed from MEGA electronics was used as shown in Figure 2.
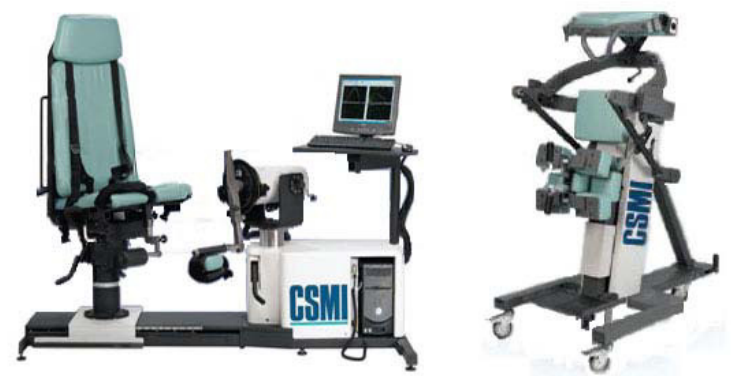

Fig. 1 . Humac Norm system

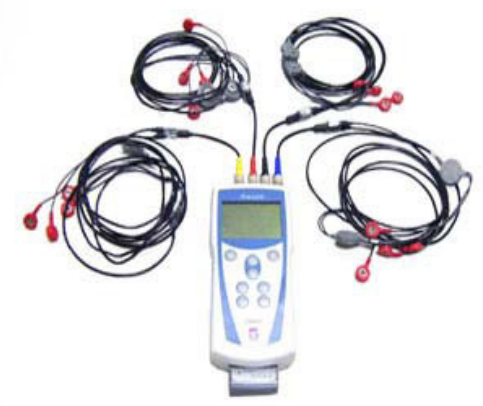

Fig. 2 ME6000 system

\subsection{Experimental design}

A two factors within-subject design was used to simulate the lifting conditions. Two loads $(25 \%, 50 \%$ MVC) and three extension angular velocity $(0,15,30$ degree/s) were independent variables. The stationarity level of EMG signals from each condition was the dependent variable.

\subsection{Procedure}

Subjects were asked to perform a simple stretching exercise to help loosen their back muscles. After the skin on the back was shaved, abraded and cleaned with alcohol, the electrodes were positioned bilaterally on the erector spinae at the L3 level $[5,10]$. Two pairs of electrodes were positioned over the belly of the erector spinae, approximately $5 \mathrm{~cm}$ apart from the center line of the spine at the L3 level.

Subjects stood upright on the Cybex system with the fixed chest, hip and legs. A maximum voluntary contraction (MVC) was performed three times for five seconds. After a five minute break, they were asked to perform isometric and isokinetic exercises. Subjects were instructed to maintain each load by monitoring the level of load themselves to minimize the effect of load variations during exertion. After each task, the subjects rested for five minutes to minimize the fatiguing effects. The sampling frequency was $1020 \mathrm{~Hz}$.

\subsection{Data Analysis}

The EMG signals were recorded during a sustained contraction with isometric and isokinetic contractions. Each signal collected from each condition was divided into time intervals by $1000 \mathrm{~ms}$ window size. Then each time interval was tested to know whether or not it was stationary by using two tests: a reverse arrangements test and a modified reverse arrangements test [9]. All data were processed using the MATLAB 2010b program and SAS 9.12 was used for statistical analysis.

\section{Results}

\subsection{The stationarity of electromyographic signals}

The stationarity of EMG signals is shown in Table 1. In the reverse arrangements test (RA test), almost all signals were stationary regardless of any conditions of load and trunk extension angular velocity. However, in the modified reverse arrangements test (MRA test), the EMG signal stationarity decreased as the trunk extension angular velocity increased. In particular, the stationarity of EMG signals in the 30 degree/s angular velocity were much lower than the 15 degree/s condition in the MRA test. 


\subsection{The effect of factors on signal stationarity}

An analysis of variance (ANOVA) was used to analyze the effect of load and trunk angular velocity on the stationarity of EMG signals. The results are shown in Table 2. In the results, there is no significance for both load and trunk angular velocity at the 0.05 level in the RA test. However, in the MRA test, the trunk angular velocity was statistically significant at the 0.05 level in the MRA test.

In post-hoc analysis as shown in Figure 3, the levels of the trunk angular velocity were divided into two groups. 0 and 15 degree/s angular velocity were grouped together by using Tukey's HSD test, whereas $30 \mathrm{degree/s}$ angular velocity showed was independent of the other angular velocity.

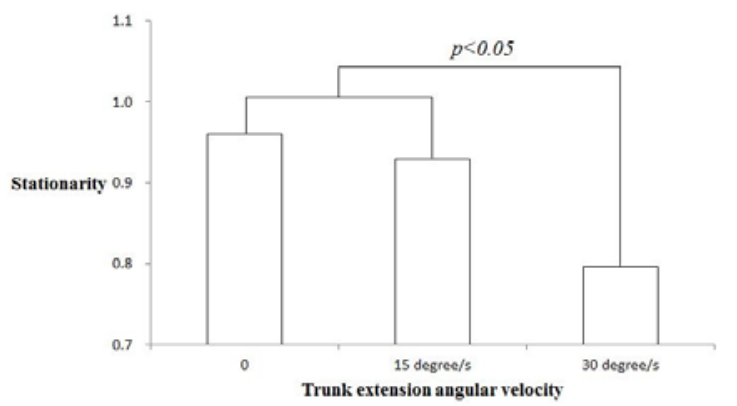

Fig. 3 Post-hoc analysis according to the trunk extension angular velocity

Table 1

The signal stationarity according to the trunk extension angular velocity and load

\begin{tabular}{|c|c|c|c|}
\hline \multirow{2}{*}{ Trunk extension angular velocity } & \multirow{2}{*}{ Load } & \multicolumn{2}{|c|}{ Stationary test } \\
\hline & & RA test & MRA test \\
\hline \multirow[t]{2}{*}{0 (isometric contraction) } & $25 \% \mathrm{MVC}$ & $1.00(0.000)$ & $0.96(0.062)$ \\
\hline & $50 \% \mathrm{MVC}$ & $1.00(0.008)$ & $0.96(0.078)$ \\
\hline \multirow[t]{2}{*}{15 degree/s } & $25 \% \mathrm{MVC}$ & $0.99(0.020)$ & $0.92(0.071)$ \\
\hline & $50 \% \mathrm{MVC}$ & $1.00(0.000)$ & $0.94(0.076)$ \\
\hline \multirow[t]{2}{*}{30 degree/s } & $25 \% \mathrm{MVC}$ & $1.00(0.000)$ & $0.87(0.088)$ \\
\hline & $50 \% \mathrm{MVC}$ & $1.00(0.000)$ & $0.72(0.232)$ \\
\hline
\end{tabular}

( ): standard deviation

Table 2

The ANOVA results of the trunk extension angular velocity and load

\begin{tabular}{|c|c|c|c|c|}
\hline \multirow{2}{*}{ Source } & \multicolumn{2}{|c|}{ RA test } & \multicolumn{2}{|c|}{ MRA test } \\
\hline & $\mathrm{F}$ & p-value & $\mathrm{F}$ & $\mathrm{p}$-value \\
\hline Trunk extension angular velocity (TE) & 0.90 & 0.4136 & 19.11 & $0.0221 *$ \\
\hline Load (L) & 1.11 & 0.3696 & 1.22 & 0.3505 \\
\hline $\mathrm{TE} \times \mathrm{L}$ & 0.90 & 0.4136 & 1.09 & 0.3724 \\
\hline
\end{tabular}

$* \mathrm{p}<0.05$ 


\section{Discussion}

Many researchers have tried to satisfy the assumption of Fourier transform through the stationary test in various conditions. Shankar et al (1989) reported that $96 \%$ of EMG signals were stationary at the low level torque and various angular velocities, whereas Beck et al. (2005) reported all EMG signals were nonstationary at the $180 \mathrm{degree} / \mathrm{s}$ angular velocity during maximal concentric contraction. However, the limited amount of research about the stationarity during isokinetic contraction meant that it was very difficult to report the level of angular velocity that could guarantee the stationarity of EMG signal. Therefore, we used various experimental conditions such as 1000 -ms window size, two levels of load $(25 \%, 50 \%$ MVC) and three levels of angular velocity $(0,15,30$ degree/s). At given conditions, almost $100 \%$ and 72 $96 \%$ of EMG signals were stationary when using the RA test and the MRA test, respectively. These results indicated that selection of stationary test techniques could be critical to accurately assess the signal stationarity.

Many researchers have agreed that the EMG signal will be nonstationary in dynamic contraction. However, this study showed that the signal stationarity during low level isokinetic contraction was not statistically different from the signals stationarity during isometric contraction. In particular, at 30 degree/s angular velocity, the stationarity of EMG signals was very low in MRA test, whereas it was high in RA test.

\section{Conclusion}

We investigated the stationarity of electromyographic signals of erector spinae muscle during isokinetic contraction. In the results, the signal stationarity decreased as trunk extension angular velocity increased as much as $30 \mathrm{degree} / \mathrm{s}$. Therefore, EMG signals collected from the condition of over 30 degree/s trunk angular velocity needs to be carefully interpreted due to the nonstationarity observed in this study.

\section{References}

[1] M. Bilodeau, M. Cincera, B.A. Arsenault and D. Gravel, Normality and stationarity of EMG signals of elbow flexor muscles during ramp and step isometric contractions, Journal of Electromyography and Kinesiology 7(2) (1997), 87-96.

[2] S. Blanco, H. Garcia, R. Quian, Q.R. Quian, L. Romanelli and O.A. Rosso, Stationarity of the EEG series, IEEE Engineering in Medicine and Biology 14 (1995), 395-399.

[3] Y. Cho and J. Kim, The effect of the signal stationarity on the EMG frequency analysis, Journal of the Ergonomics Society of Korea 29(2) (2010), 183-188.

[4] P. Coorevits, L. Danneels, D. Cambier, H. Ramon, H. Druyts, S.J. Karlsson, G.D. Moor and G. Vanderstraeten, Correlations between short-time Fourier- and continuous wavelet transforms in the analysis of localized back and hip muscle fatigue during isometric contractions, Journal of Electromyography and Kinesiology 18 (2008), 637-644.

[5] J.R. Cram, G.S. Kasman and J. Holtz, Introduction to Surface Electromyography, first ed. Aspen Publishers, Gaitherburg, Md, 1998.

[6] G.F. Inbar and A.E. Noujaim, On surface EMG spectral characterization and its application to diagnostic classification, IEEE Transactions on Biomedical Engineering 31 (1984), 597-604.

[7] J.Y. Kim, M.C. Jung and J.M. Haight, The sensitivity of autoregressive model coefficient in quantification of trunk muscle fatigue during a sustained isometric contraction, International Journal of Industrial Ergonomics 35(4) (2005), 321-330.

[8] A.V. Oppenheim and R.W. Schafer, Discrete-time signal processing, second ed. Prentice Hall, Upper Saddle River, 1999.

[9] S. Siegel and Jr N.J. Castellan, Nonparametric statistics for the behavioral sciences, second ed. McGraw-Hill, New York, 1988.

[10] G.L. Soderberg, Selected Topics in Surface electromyography for Use in the Occupational Setting: Expert Perspectives, Rockville, Md: US Department of Health and Human Services, Public Health Service, 1992. 